\title{
Path Planning Method for Electric Vehicles Based on Freeway Network
}

\author{
Qichao Liu $\mathbb{D}^{1,2,3}$ Wei Wang $\mathbb{D}^{1,2,3}$ and Xuedong Hua $\mathbb{D}^{1,2,3}$ \\ ${ }^{1}$ Jiangsu Key Laboratory of Urban ITS, Southeast University, Nanjing 211189, China \\ ${ }^{2}$ Jiangsu Province Collaborative Innovation Center of Modern Urban Traffic Technologies, Southeast University, Nanjing 211189, \\ China \\ ${ }^{3}$ School of Transportation, Southeast University, Nanjing 211189, China
}

Correspondence should be addressed to Wei Wang; wangwei@seu.edu.cn

Received 20 May 2021; Revised 1 September 2021; Accepted 6 October 2021; Published 18 October 2021

Academic Editor: Giuseppe Musolino

Copyright (c) 2021 Qichao Liu et al. This is an open access article distributed under the Creative Commons Attribution License, which permits unrestricted use, distribution, and reproduction in any medium, provided the original work is properly cited.

\begin{abstract}
Recently, electric vehicles (EVs) have received more and more attention, but the problem of the insufficient range is still the main reason that hinders electric vehicles to travel long distances. Under the premise of the battery capacity without technological innovation, the path planning method can ensure the safety and efficiency of electric vehicles in long-distance travel. This paper studies the actual freeway network to optimize the vehicle driving path and give the charging strategy based on the shortest travel time of a single vehicle. In this paper, a path and charging strategy planning model is proposed. In this model, the shortest travel time of a single vehicle is taken as the objective function, and the state of charging equipment in the actual road network and the safe electric quantity are considered as constraints. And the genetic algorithm is used to solve the model. Through case analysis, the rationality and optimization efficiency of the model proposed in this paper are verified. Finally, the sensitivity analysis of the three parameters of traffic volume, temperature, and travel speed is carried out with the Shanghai-Nanjing freeway network. The experimental results can get the nodes with the highest service pressure in the network, which can provide a theoretical basis for charging nodes' expansion in the freeway network in the future.
\end{abstract}

\section{Introduction}

With the intensification of environmental pollution and energy shortages, energy conservation and environmental protection have become a major theme of the current society. At the same time, with the vigorous development of the automobile industry, traditional fuel vehicles have the disadvantages of relying on a large amount of petroleum resources and emitting large exhaust gas pollution. Therefore, all countries are actively developing the new energy automobile industry.

Currently, the new energy vehicle market share increased year by year; however, the electric vehicle battery technology is still immature. In the highway scene, electric vehicles have obvious shortcomings compared to fuel vehicles. Most electric vehicle owners have the problem of "endurance anxiety". Under this background, the battery life of electric vehicles has become the main direction of current scholars.

In combination with the actual situation, if the owners of pure electric vehicles travel a long distance, the phenomenon of in-journey charging will certainly occur. The owners of pure electric vehicles can only choose the service area with charging piles to park and charge on the freeway; then, they may need to queue up when they arrive at the service area, which leads to the delay in travel time. In summary, there are two main difficulties that electric vehicles face when driving on freeways: one is the battery life of electric vehicles; the other is the delay caused by charging.

At present, researches on solving the above two problems are mainly focused on three directions: first, improve the technology in the field of battery to improve the endurance mileage of vehicles; second, increase the number of charging stations to improve the convenience of $\mathrm{EV}$ 
charging; third, choose the appropriate driving strategy to reduce the consumption of electricity. The following three paragraphs describe the current research of the above three research directions, respectively.

Considering that during the driving process of an electric vehicle, its energy consumption has a great relationship with the driving state of the vehicle, so in order to accurately describe the changing trend of its energy consumption, many scholars have done a lot of research on this. Genikomsaki [1] proposed a simulation model for calculating the energy consumption of electric vehicles based on path planning applications. The model uses dynamic methods to simulate the energy recovery capacity of electric vehicles. The output of the simulation model is compared with the output of FASTSim based on real data. The results show that the established model achieves a reasonable balance between expression efficiency and calculation efficiency and can be used to estimate energy consumption in the path of electric vehicles. $\mathrm{Xu}$ et al. [2] think that, in a large network environment, the whole system simulation tool to predict the energy consumption will encounter scaling problems, so this paper proposes a new method for estimating the power consumption of the electric vehicle and the change of the vehicle running conditions, under the support of large-scale traffic network of energy estimates that the modeling method can be used to evaluate various simulation traffic network level of the study of energy use. Yi et al. [3] proposed a real-time adaptive multiresolution energy consumption estimation system for electric vehicles. The concept of "networked vehicles" was adopted to achieve a more reliable energy estimation of travel level by sharing the perceived environmental information. Wager et al. [4] found that electric vehicles use loads when driving on expressways, and since the safety margin of fast charging and discharging reduces the charging capacity, the influence of these parameters on the energy consumption and range of electric vehicles was studied. Zhang et al. [5] collected data on the driving conditions of electric vehicles on urban roads, analyzed the characteristics of the instantaneous speed, acceleration, and charge state of microscopic driving parameters, and established energy consumption estimation models in different operating modes from a statistical point of view. Cauwer et al. [6] established three energy consumption prediction models based on the vehicle dynamics model based on measured data and compared the prediction accuracy of the three models. Croce et al. [7] proposed an energy consumption estimation model that can estimate the SOC of electric vehicles in combination with traffic flow theory, dynamic model, and information and communication technology. Croce et al. [8] proposed a transportation service optimization decision-making framework to reduce energy consumption. Liu et al. [9] established the charging strategy model of lithium electronic batteries by using the multiobjective constrained nonlinear optimization method. The model takes battery health, charging time, and energy conversion efficiency as the charging objectives, and the model can propose an appropriate charging strategy. Liu et al. [10] proposed a constrained multiobjective optimization framework to realize economical battery charging management. Liu et al. [11] proposed a model-based charging mode optimization strategy, considering the key parameters such as charging speed, energy conversion efficiency, and temperature change.

At the same time, during the driving process of an electric vehicle, it is usually necessary to consider the problem of mileage anxiety, so it is necessary to estimate the remaining battery capacity, especially in the process of planning the path of the electric vehicle. SOC (State of Charge) is used to reflect the remaining capacity of the battery. It is numerically defined as the ratio of the remaining capacity to the battery capacity, which is usually expressed as a percentage. Its value range is $0-1$. When $\mathrm{SOC}=0$, it means the battery is completely discharged; when $\mathrm{SOC}=1$, it means the battery is fully charged. Paschero et al. [12] introduced a new battery model consisting of three electric dipoles, which only rely on voltage measurement and a given current curve for parameter estimation and use Kalman filtering to estimate it. Dong et al. [13] and others considered the battery management system and established a state-of-charge (SOC) estimation method based on extended Kalman filter (EKF) and adaptive extended Kalman filter (AEKF) and performed it under federal urban driving conditions. Li et al. [14] proposed a model-based sequential Monte Carlo filter combined with adaptive control to determine the battery SOC and a new method of electrical impedance and verify its applicability. Baek et al. [15] proposed a general method to model the path, vehicle, and battery, predict or optimize the operating range of electric vehicles, and alleviate mileage anxiety. Baek et al. [16] implemented an electric vehicle simulator based on the electric vehicle powertrain model and nonlinear battery model. Chen et al. [17] realized early electric vehicle simulation by defining the System CAMS framework, which models both physical and mechanical evolution, energy flow, and environmental characteristics.

For electric vehicle charging planning, on the one hand, it is planning for charging stations, usually studying the location and capacity of charging stations; on the other hand, it is planning for electric vehicle paths, which can be divided into static planning and real-time charging navigation. Electric vehicle charging station planning is mainly based on forecasting the demand for electric vehicles and analyzing their location and capacity. Arslan et al. [18] considered the location problem of the combination of electric vehicles and plug-in hybrid vehicles, maximized the mileage of the vehicle under the condition of cost constraints, and used the Benders decomposition algorithm to solve it. Taking into account the uncertainty of random traffic flow, $\mathrm{Wu}$ et al. [19] established a random traffic capture and positioning model for public fast-charging station planning. He et al. [20] established a two-layer programming model in which the lower layer is the user path selection constrained by EV mileage and the upper layer is the optimal charging station location in order to determine the optimal charging station location. He [21] discussed how to optimize the location of public charging stations for electric vehicles in the road network, considering the driver's spontaneous adjustment and the interaction between travel 
and charging decisions. In the case of uncertain mileage, Boujelben [22] proposed two models based on stochastic programming to deal with the resulting random fastcharging site selection problem. The first model maximizes the expected traffic coverage of the network, and the second is based on joint chance constraints. Chen [23] proposed a two-layer mathematical model considering the balance of path selection and charging waiting time for the optimal location and capacity of charging stations. It is based on the calculation of facility construction and electric vehicle drivers' journey and waiting time on the network. The goal is to minimize the total cost and apply it to the network to evaluate its robustness. Kong et al. [24] comprehensively considered the influence of factors such as operators, drivers, vehicles, traffic flow, and power grids for modeling.

The current research on electric vehicle charging strategy focuses on the impact of electric vehicle charging on the grid load and then gives the optimal charging strategy. $M$ Schücking et al. [25] studied the use of electric vehicles to replace traditional fuel vehicles in commercial fleets and analyzed five different strategies given in the past. The research results show that the combination of conventional and fast charging can achieve higher mileage. Dong et al. [26] proposed a pricing strategy for fast charging stations for electric vehicles in order to determine the pricing plan for voltage control in the distribution network. Through the analysis of the user's travel characteristics, a simulation model for the mobility of electric vehicles was established. Yi et al. [27] proposed a strategy for the selection of charging stations and the amount of charging based on individual users, considering their daily itinerary and limitations of charging facilities. Elgarej et al. [28] proposed an electric vehicle path planning system based on distributed ant colony algorithm. By proposing a group of nearest charging stations, the total path of the electric vehicle to the destination is minimized. Based on the time-sharing leasing mode of electric vehicles, Guo et al. [29] discussed the configuration of the electric vehicle service station in intercity traffic considering user experience and multipath allowed by each destination pair. Wang et al. [30] established an extended network structure to build an effective charging strategy model for electric vehicle drivers. Taking a highway network as an example, the results show that the strategy can solve large-scale problems within the optimality gap of less than 5\%. Liu et al. [31] studied how to make an appointment charging strategy to ensure that electric vehicles can charge with shorter charging time and lower charging cost when charging requirements are put forward. Li et al. [32] communicated vehicles with infrastructure components and real-time transmission of information, in order to find the best route. Taking an actual road network in Charleston, South Carolina, as the simulation road network, the results show that the electric vehicle network can not only reduce the total driving time and energy consumption but also reduce the charging power and the corresponding cost, so as to significantly alleviate the mileage anxiety of electric vehicle drivers. Based on the charging demand information, Wang et al. [33] put forward two kinds of path guidance strategies from different angles to provide effective charging stations and the corresponding route selection. Both of these strategies can ensure reaching the selected charging station in the time-varying road network.

In previous studies, the research object is usually a virtual road network, and the research focus is usually battery technology and charging station layout. On the premise of the bottleneck period of battery technology development, in order to solve the mileage anxiety of electric vehicles on freeways, we consider using the method of vehicle path planning to reduce the traffic pressure of electric vehicles on freeways.

The main contributions in this paper are summarized: (1) we propose a path planning model that can provide the vehicle's driving path and charging strategy and prove that the path and charging strategy provided by the model can shorten the travel time; (2) we conduct sensitivity analysis for several important parameters and analyze the impact of important parameters on the road network and driving efficiency; (3) based on the results of the sensitivity analysis, we proposed improvement measures for the freeway network.

The rest of the pare is organized as follows. In Section 2, we propose a path planning model and solve it. In Section 3, we conducted case studies and proved the rationality of our model.

In Section 4, we take the Shanghai-Nanjing freeway network as the research object and analyze the retrograde sensitivity. Finally, we summarize all the experimental results.

\section{Problem Modeling}

Consider a directed network $G(N, A)$, where $N$ is the set of nodes and $\mathrm{A}$ is the set of links. The directed network is built according to the actual freeway network. In the freeway network, the path planning problem of electric vehicles is to find the reliable shortest path, the remaining enough battery power, and the shortest travel time. There are several basic assumptions in this paper: (1) vehicles in the freeway network do not interfere with each other and travel at a given speed at a constant speed; (2) vehicles must be charged on the freeway network.

2.1. Objective Function. The main optimization objective of the model in this paper is to improve the traffic efficiency of electric vehicles, so the objective function is divided into two parts:

$$
\min J=\min (t-c h),
$$

where $t$ is the travel time of the electric vehicle, min; ch is the node number of the EV is selected for charging. The first part is the shortest travel time of vehicles under the freeway network. The second part ensures that the vehicle can be charged as close as possible to the destination node $d$, which can avoid the unreasonable charging nodes of the electric vehicle.

The reliable travel time of path $P$ between the origin node $o$ and destination node $d$ can be formulated as a linear 
combination of the actual vehicle travel time, charging queuing time, and actual charging time:

$$
t=t_{d}+t_{w}^{c h}+t_{c}^{c h},
$$

where $t_{d}$ is the actual travel time which is the travel time of vehicles on the links, $\min ; t_{w}^{c h}$ is the queuing time of vehicle at the ch node, min; $t_{c}^{c h}$ is the time for the vehicle to be fully charged at the ch node, min.

Because the vehicle is traveling at a constant velocity on the highway, the travel time of the vehicle on the link can be calculated by

$$
t_{d}=\frac{l_{1}+l_{n-1}}{v_{c}}+\frac{\sum_{i=2}^{n-2} l_{i}}{v_{h}} .
$$

Considering the speed of urban expressways is different from the speed of freeways, this paper divides the actual travel time into two parts: urban expressway travel time and freeway travel time, where $l_{1}$ and $l_{n-1}$ represent the length of the urban freeway link in the directed network, $\mathrm{km}$; $l_{i}$ is the length of freeway link in the directed network, $\mathrm{km} ; v_{c}$ is the constant velocity of the urban expressway, $\mathrm{km} / \mathrm{h} ; v_{h}$ is the constant velocity of the freeway, $\mathrm{km} / \mathrm{h}$.

The waiting time for the vehicle can be calculated by formulas (4)-(6):

$$
t_{w}^{q, c h}=t_{s}^{q, c h}-t_{a}^{q, c h}
$$

where $t_{s}^{c h}$ is the time when the vehicle $q$ starts charging at a node $\mathrm{ch}, \mathrm{min} ; t_{a}^{c h}$ is the time when the vehicle $q$ arrives at the node $c h$, min. According to the arrival time of the vehicle, when the vehicle arrives and the charging pile is free, the vehicle can directly start charging. If there is no free charging pile when the vehicle arrives, it needs to wait until the charging pile is free as shown in

$$
t_{w}^{q, c h}=\left\{\begin{array}{l}
t_{s_{\min }}^{q, c h}, t_{a}^{q, c h} \leq t_{s_{\min }^{q, c h}}^{q, c h} \\
t_{a}^{q, c h}, t_{a}^{q, c h}>t_{s_{\min }}^{q, c h}
\end{array}\right.
$$

The starting charging time of the vehicle can be calculated by

$$
t_{s_{\min }}^{q, c h}=\min \left\{t_{s}^{q-N_{c h}, c h}+t_{c}^{q-N_{c h}}, t_{s}^{q-N_{c h}+1, c h}+t_{c}^{q-N_{c h}+1}, \ldots, t_{s}^{q-1, c h}+t_{c}^{q-1}\right\},
$$

where $N_{c h}$ is the number of the charging piles of node ch. Since the use of charging piles conforms to the principle of first come, first served, the earliest charging time of vehicle $q$ is determined by vehicles $\left[q-N_{c h}, q-N_{c h}+1, \ldots, q-1\right]$. $t_{s}^{q, c h} \min$ is the earliest charging time of vehicle $q$.

The charging time of the vehicle is calculated according to formulas (7) and (8):

$$
t_{c}^{c h}=\frac{\left(S O C_{u p}-S O C_{c h}\right)}{\eta P_{c}} E_{0},
$$

where $E_{0}$ is the rated capacity of the on-board battery of an electric vehicle, kwh; $\eta$ is the charging efficiency, the conventional value is $0.9 ; S_{c h}$ represents the remaining SOC when the vehicle arrives at the node ch; $\mathrm{SOC}_{u p}$ is the state of the on-board battery after charging. When it is fully charged, $S O C_{u p}$ is $1 . P_{c}$ represents the charging power of the electric vehicle, $\mathrm{kw}$, where the SOC when reaching the node ch can be obtained by

$$
S O C_{c h}=\left(S O C_{o}-S O C_{c o n s}\right),
$$

where $S_{0} C_{o}$ is the SOC of the vehicle at the starting point $\mathrm{o} ; S O C_{c o n s}$ is the SOC consumed from the starting point o to the charging node $c h$. The SOC consumed can be calculated according to formula (9), as follows:

$$
S O C_{\text {cons }}=\frac{y\left(v_{c}\right) l_{1}+y\left(v_{h}\right) \sum_{i=2}^{c h} l_{i}}{E_{0}},
$$

where $y$ is the energy consumption at different speeds, kwh/ $\mathrm{km}$. In 2017, Ray Galvin used data from eight common electric vehicles to develop a multiple regression model that can describe the relationship between speed and energy consumption. The fitted curve is shown in Figure 1. We use Ray Galvin's model [34] to calculate the energy consumption of electric vehicles at different speeds, as shown in

$$
y=0.00002 v^{2}-0.0015 v+0.1331
$$

2.2. Constraints. Considering the actual situation of the electric vehicle in the freeway network, the constraint conditions of the model in this paper are shown in formulas (11)-(18).

2.2.1. Constraint for SOC. The electric vehicle cannot run out of power in the freeway network, so the SOC of the vehicle in this paper must be greater than the fixed value:

$$
\mathrm{SOC}_{s} \leq \mathrm{SOC}_{m} \leq \mathrm{SOC}_{u p}, m \in[1, n],
$$

where $\mathrm{SOC}_{s}$ is the security SOC. The SOC of the vehicle arriving at the node $\mathrm{m}$ can be calculated according to formulas (8) and (9).

2.2.2. Constraint for Charging Pile. The number of charging piles in each node is fixed, and the number of vehicles being charged at the same time cannot exceed the number of charging piles in the node:

$$
0 \leq \mu_{c h}(t) \leq w(c h)
$$

where $\mu_{c h}(t)$ denotes the number of vehicles charging in the node ch at time $\mathrm{t}$, veh; $w(c h)$ denotes the number of available charging piles of charging node $\mathrm{ch}$.

2.2.3. Constraint for Queue Length. Due to safety factors, the number of queuing vehicles at each charging node should not exceed a queuing length threshold:

$$
q l_{c h}(t) \leq q l_{\max }
$$

where $q l_{c h}(t)$ is the queuing length of the charging node ch at time $t$, veh; $q l_{\text {max }}$ is the threshold of the queue length allowed by the charging node, veh. 


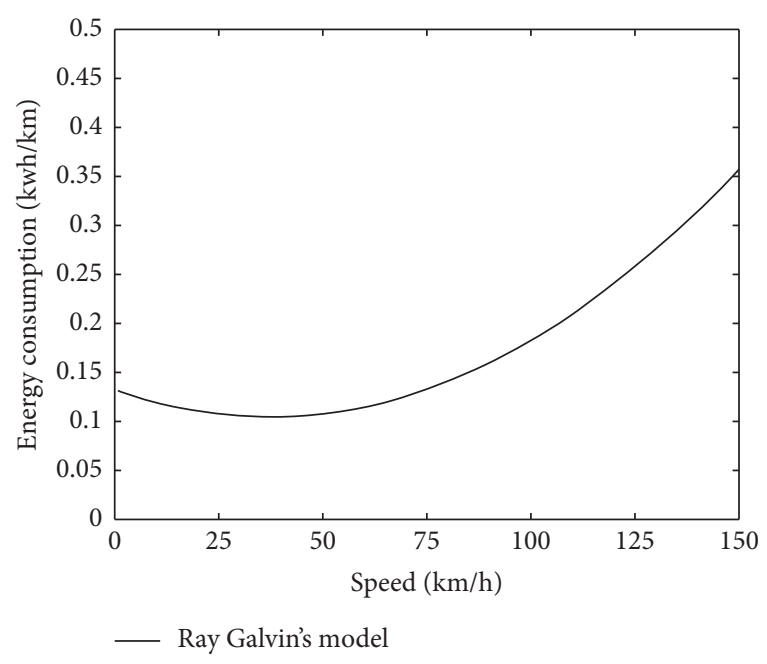

Figure 1: Ray Galvin's model.

2.2.4. Constraint for the Path Direction. In the process of building the road network, the path under the freeway network has the characteristics of gradually increasing node number. Therefore, we use the constraints of the path direction to eliminate the solutions that do not meet the characteristics of the path. Path direction constraint is

$$
s_{i+1}>s_{i}, i \in[1, n-1] \text {. }
$$

2.2.5. Constraint for Origin and Destination. In the process of solving, the origin and the destination of the vehicle are limited. The path obtained by the solution can only start from the specified origin to the specified destination:

$$
\begin{aligned}
& s_{1}=o, \\
& s_{n}=d,
\end{aligned}
$$

where o represents the specified starting point number; $d$ stands for the specified destination number.

2.2.6. Constraint for Charging Nodes. The vehicle must select the node in the path for charging, as shown in

$$
c h \in s \text {. }
$$

2.2.7. Constraint for Destination SOC. Considering that it is possible to continue driving after reaching the destination, enough remaining SOC is retained:

$$
\mathrm{SOC}_{d} \geq \mathrm{SOC}_{r}
$$

where $\mathrm{SOC}_{d}$ is the SOC of the destination and $\mathrm{SOC}_{r}$ is the residual SOC threshold.

2.3. Solution. Since the proposed model contains nonlinear constraints, the proposed model is an integer nonlinear programming problem. Considering the need to control the path and charging nodes at the same time, it is difficult to solve the problem by using the traditional shortest path model. A heuristic algorithm is often used to solve this problem. Therefore, this model was figured out based on the genetic algorithm (GA) in this paper, and the procedure is shown as follows.

Step 1. Parameter Input. Parameters include road network adjacency matrix and state parameters of each charging node. Go to Step 2.

Step 2. Randomly generate the initial path scheme and the charging scheme. Go to Step 3.

Step 3. Perform survival operations. Survival operations include selection, crossover, and mutation. Go to Step 4 .

Step 4. Calculation of Fitness. Fitness is the inverse of the objective function. The last $50 \%$ of optimal individuals will be selected to survive. Go to Step 5 .

Step 5. Termination Condition Judgment. Judge whether the termination condition is met; if so, output the optimal path and charging strategy; if not, go to Step 3.

The detailed solution process is shown in Figure 2.

\section{Plausibility Validation}

We applied the proposed model to a case analysis and compared it with a conventional path selection scheme (electric vehicles prefer the shortest path). The conventional path selection scheme is that the vehicle chooses the path with the shortest distance and selects the last charging node before the vehicle runs out of power for charging. The introduction of the case study road network, charging node status, and comparison results will be described. Figure 3 is the road network diagram of the case analysis.

The values of parameters in the case analysis are shown in Table 1.

The accuracy of the algorithm and the effect of optimization were tested by a case study. The case network is shown in the figure. Set up 12 electric vehicles to run in the case network. The starting point is node 1 . The destination is node 13 . The arrival time interval of electric vehicle conforms to the Poisson distribution.

3.1. Comparative Analysis of Path. Figure 4 is the path comparison analysis diagram, where (a) is the conventional path selection scheme and (b) is the path selection scheme of the model proposed in this paper, where path 1 is 1-3-5-8$10-12-13$, path 2 is $1-3-5-7-10-12-13$, and path 3 is $1-3-6-11-$ 13. The vehicle entering the system first will give priority to path 1 and the charging node on path 1 . When the queuing length of the charging node on path 1 is too long, the vehicle will select path 2 and the node on path 2 . When the queuing length of all paths 1 and 2 is too long, the vehicle will start to select path 3 and the node on path 3 . As can be seen from the figure, the electric vehicle chooses the same three paths 


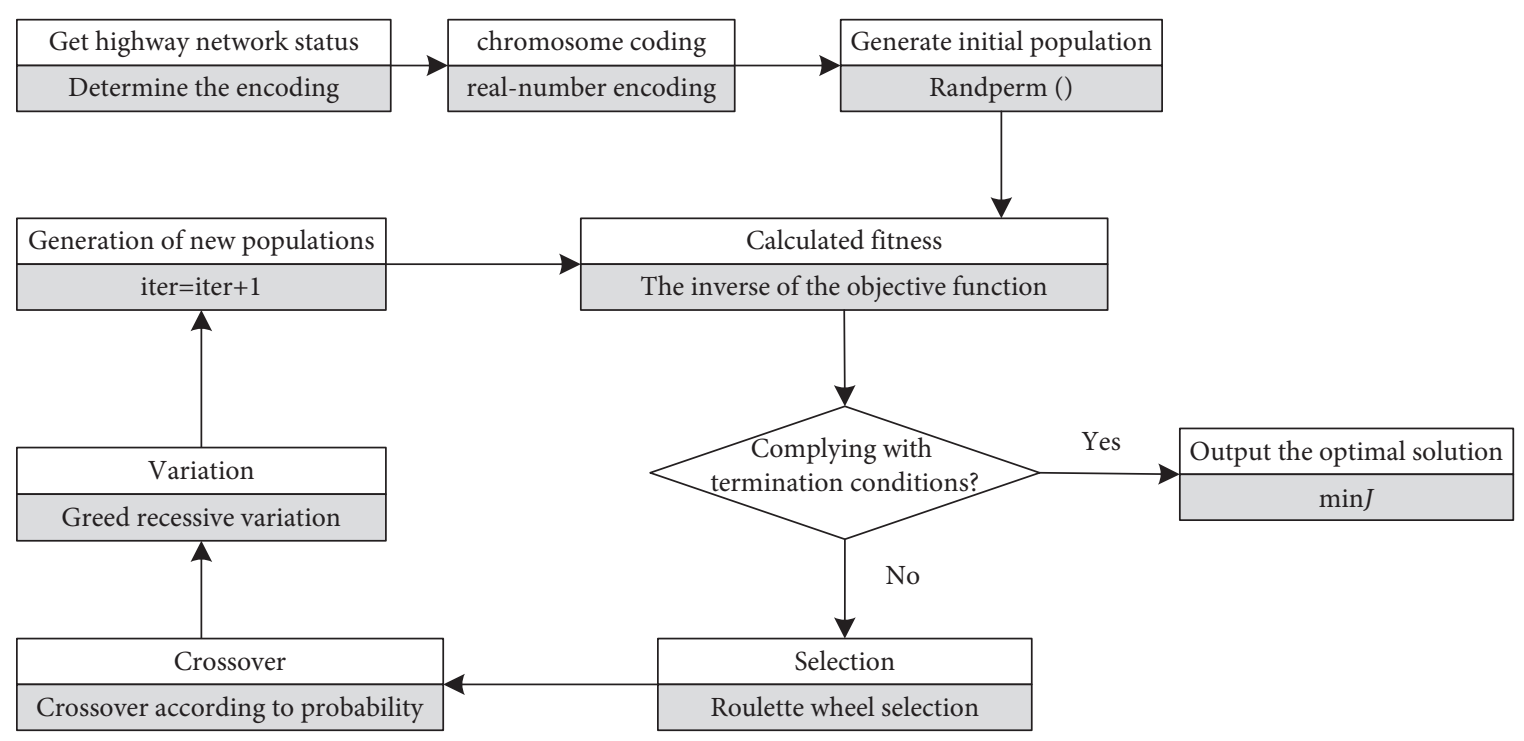

FIgURE 2: Model solution process.

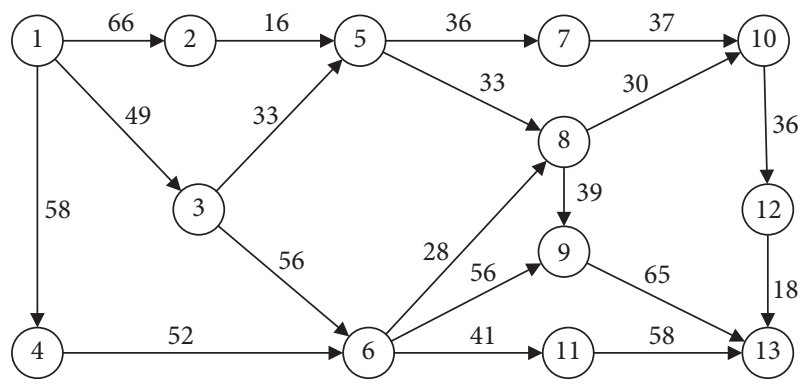

Figure 3: Case network.

TABLE 1: Case analysis parameters.

\begin{tabular}{lccc}
\hline Notion & Parameters & Value & Unit \\
\hline$v_{c}$ & Urban highway running speed & 60 & $\mathrm{~km} / \mathrm{h}$ \\
$v_{h}$ & Freeway running speed & 90 & $\mathrm{~km} / \mathrm{h}$ \\
$E_{0}$ & Rated capacity of the on-board battery & 40 & $\mathrm{kwh}$ \\
$\eta$ & Charging efficiency & 0.9 & - \\
$S O C_{u p}$ & State of the on-board battery after & 0.8 & - \\
$P_{C}$ & $\quad$ charging & 120 & $\mathrm{kw}$ \\
$S_{S O}$ & Charging power & 0.2 & - \\
$S O C_{d}$ & Security SOC & 0.2 & - \\
$\mathrm{w}$ & SOC of the destination & 2 & - \\
$q l_{\max }$ & Node charging pile number & 2 & $\mathrm{veh}$ \\
\hline
\end{tabular}

under the different path selection schemes. The difference is that there are more charging nodes in the proposed model scheme than in the conventional scheme. To sum up, the proposed model can get a reasonable driving path and charging strategy.

3.2. Comparative Analysis of Travel Time. Figure 5 shows the travel time of 12 electric vehicles under different schemes. As shown in the figure, the average travel time of the vehicle decreased from 4.25 hours to 3.97 hours, reducing the travel time by $6.5 \%$. The reason is that the travel time is changed by changing the path or charging node of the vehicle through the control strategy, and the late-arriving vehicle chooses the charging node in front of it to charge to avoid queuing.

3.3. Comparative Analysis of Queuing Time. Figure 6 illustrates the queuing time of vehicles before and after optimization. Scatter point is the queuing time of a single vehicle. It can be seen from the figure that the queuing time at the node after optimization decreases compared with that before optimization. From the trend line of queuing time, the average queuing time shows a downward trend. Before optimization, the average queuing time was 0.23 hours, but after adopting the scheme proposed in this paper, the average queuing time dropped to 0.08 hours, reducing the queuing time by about $65 \%$.

As can be seen from Figure 7, after optimization, the proportion of vehicles queuing decreased significantly, from the original $50 \%$ to $33.3 \%$, and the number of vehicles queuing changed from 6 to 4 .

It can be seen from the above comparative experiments that the path planning method proposed in this paper can provide a reasonable path and charging strategy. Compared with the conventional path planning method, the method proposed in this paper can effectively improve the traffic efficiency of electric vehicles on freeways.

\section{Sensitivity Analysis}

To further analyze the adaptability of the proposed path optimization method, sensitivity analysis of critical parameters was carried out to explore the influence of driving efficiency and highway charging equipment under the actual freeway network. 


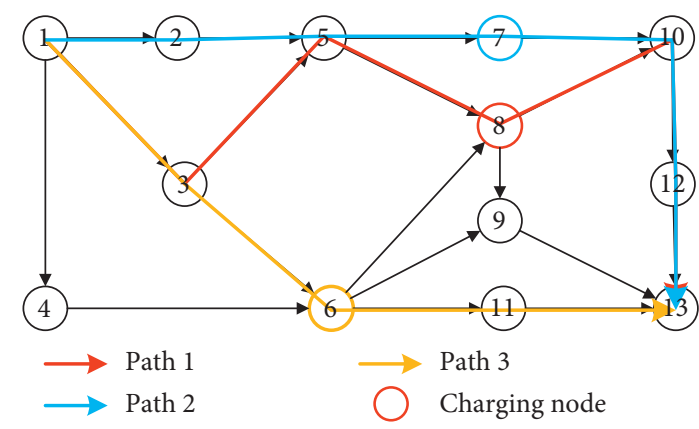

(a)

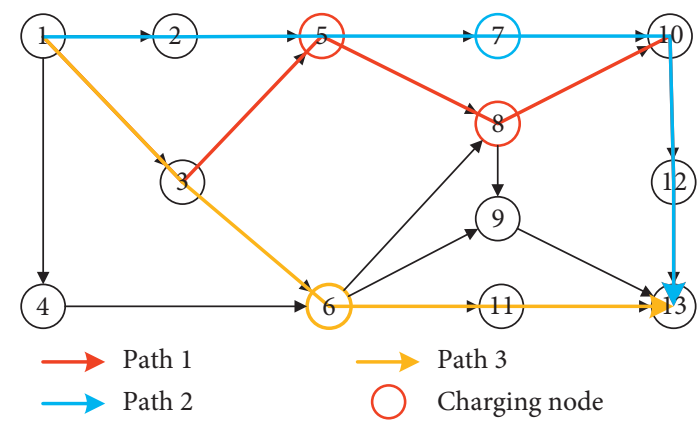

(b)

Figure 4: Path contrast.

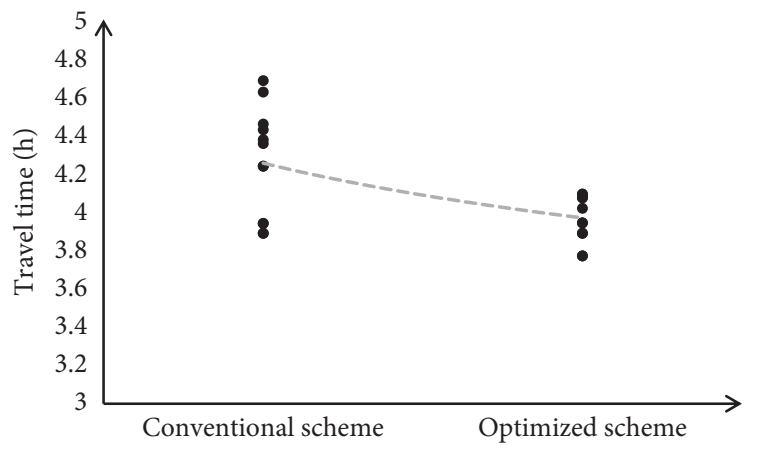

Figure 5: Average travel time contrast.

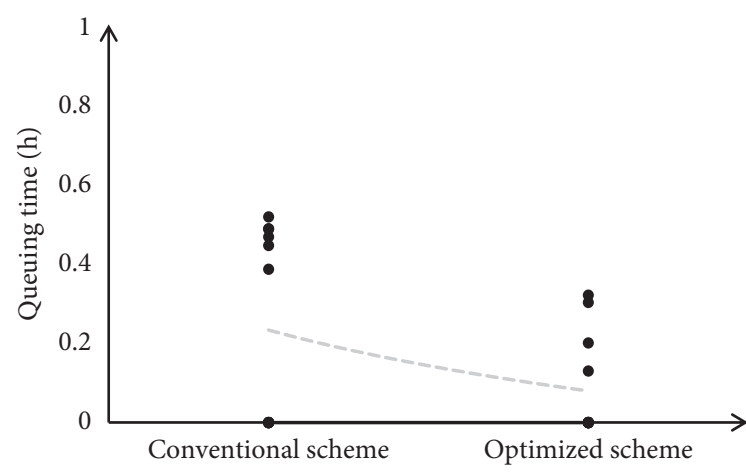

Figure 6: Average queuing time contrast.

4.1. Construction of Actual Freeway Network. In this paper, Shanghai and Nanjing are selected as the origin and destination of the freeway network, respectively, to build the Shanghai-Nanjing freeway network. The steps are as follows:

(1) Node selection: Select the service area in the freeway as the node. The selected nodes are shown in Table 2.

(2) Data collection: The collected data include node spacing, number of charging piles on nodes, and charging power of charging piles.

(3) Network topology: The actual freeway network is abstracted into the form of a directed network graph and the adjacency matrix is established.

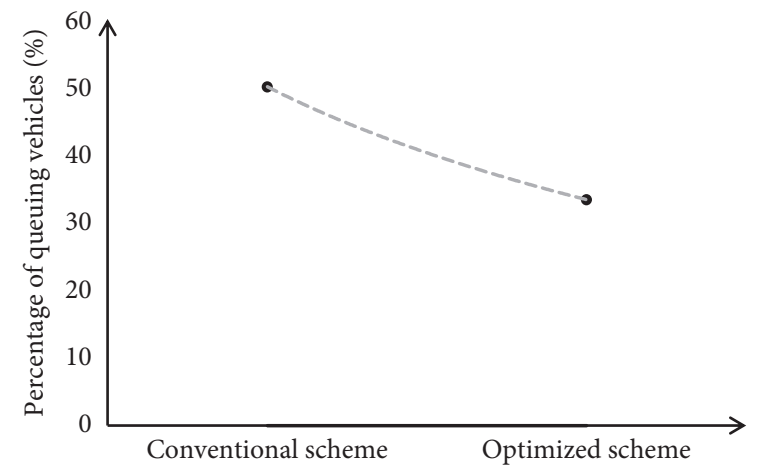

Figure 7: Percentage of queuing vehicles contrast.

The freeway network finally established is shown in Figure 8 , and the freeway network topology is shown in Figure 9.

The environmental data of the Shanghai-Nanjing Freeway Network were collected to determine the parameter input in the simulation environment. The parameter values are shown in Table 3.

4.2. Effect of Traffic Volume. The volume of electric vehicles gradually increases from low volume to high volume; other parameters are consistent with Table 3. Figures 10(a), 10(b), and $10(\mathrm{c})$ are, respectively, vehicle trajectories under low volume, medium volume, and high volume. In the case of low traffic volume, vehicles in the freeway network choose 2 paths, increasing to 4 in the case of medium traffic volume, and 7 in the case of high traffic volume. The number of alternative paths gradually increases, and the number of selected charging nodes also increases. It can be seen that the total distances of the four optional paths are not significantly different and the selection of charging nodes is relatively flexible under the condition of medium traffic volume. In the case of high traffic volume, the driving distance of some vehicles will increase considerably, because the vehicles can take a long way around to the destination in a time equivalent to or less than that of queuing at the nodes of the short path. 
TABLE 2: Network node sequence.

\begin{tabular}{|c|c|c|c|}
\hline Node number & Node & Node number & Node \\
\hline 1 & Shanghai & 20 & Jiangyin bridge service area \\
\hline 2 & Gangyan service area & 21 & Yixing service area \\
\hline 3 & Dianshanhu service area & 22 & Taihu (Yixing) service area \\
\hline 4 & Shaxi service area & 23 & Tianmuhu service area \\
\hline 5 & Chongqi bridge service area & 24 & Guocun service area \\
\hline 6 & Qilin service area & 25 & Guangling service area \\
\hline 7 & Shipai service area & 26 & Xinqiao service area \\
\hline 8 & Baiyanghu service area & 27 & Furong service area \\
\hline 9 & Pingwang service area & 28 & Shouxihu service area \\
\hline 10 & Xianfeng service area & 29 & Yizheng service area \\
\hline 11 & Yangchenghu service area & 30 & Rongbing service area \\
\hline 12 & Taihu (Huzhou) service area & 31 & Gehu service area \\
\hline 13 & Rugao service area & 32 & Donglushan service area \\
\hline 14 & Yangcheng beihu service area & 33 & Xianrenshan service area \\
\hline 15 & Yangcheng Xihu service area & 34 & Huanglishu service area \\
\hline 16 & Meicun service area & 35 & Siqiao service area \\
\hline 17 & Baimi service area & 36 & Jiangning service area \\
\hline 18 & Yanqiao service area & 37 & Nanjing \\
\hline 19 & Wanshandang service area & & \\
\hline
\end{tabular}

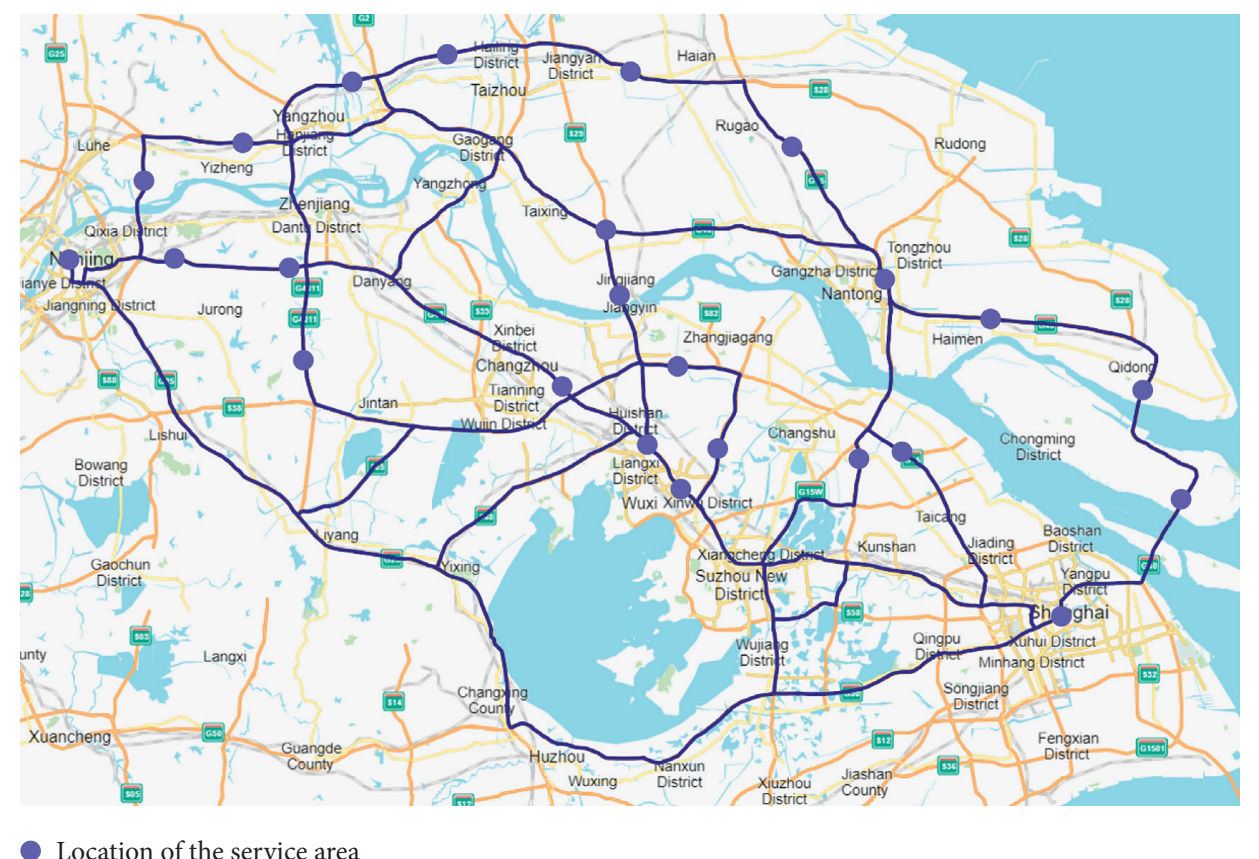

Figure 8: Actual freeway network.

The red dots in Figure 11 are all charging nodes in the freeway network, and the thermal circle covers the charging points selected on the vehicle driving path. Figures 11(a), 11(b), and 11(c) are, respectively, node utilization under low volume, medium volume, and high volume. As shown in Figure 11, charging piles in the Furong service area and Yanqiao service area have the highest utilization under different flow rates. In the future, it can be considered to add charging piles in the above two service areas.

Figure 12 shows the distribution of charging nodes in use at different volumes. The warmer the color of the heat map is, the higher the utilization of charging nodes in this area.
Figures 11 and 12 are all heat maps. Figure 11 focuses on the impact degree of a single node, and Figure 12 focuses on the impact degree in spatial distribution. As can be seen from the figure, the charging node utilization in Wuxi and Changzhou is relatively high. When the volume rises to a high volume, charging nodes in Yixing and Taixing areas also begin to be fully utilized. To sum up, Wuxi and Changzhou are the areas where charging behaviors occur most frequently on the Shanghai-Nanjing freeway. In the future, the number of charging piles can be increased in Wuxi and Changzhou, which can greatly improve the alleviation of the phenomenon of queuing for charging pure electric vehicles. 


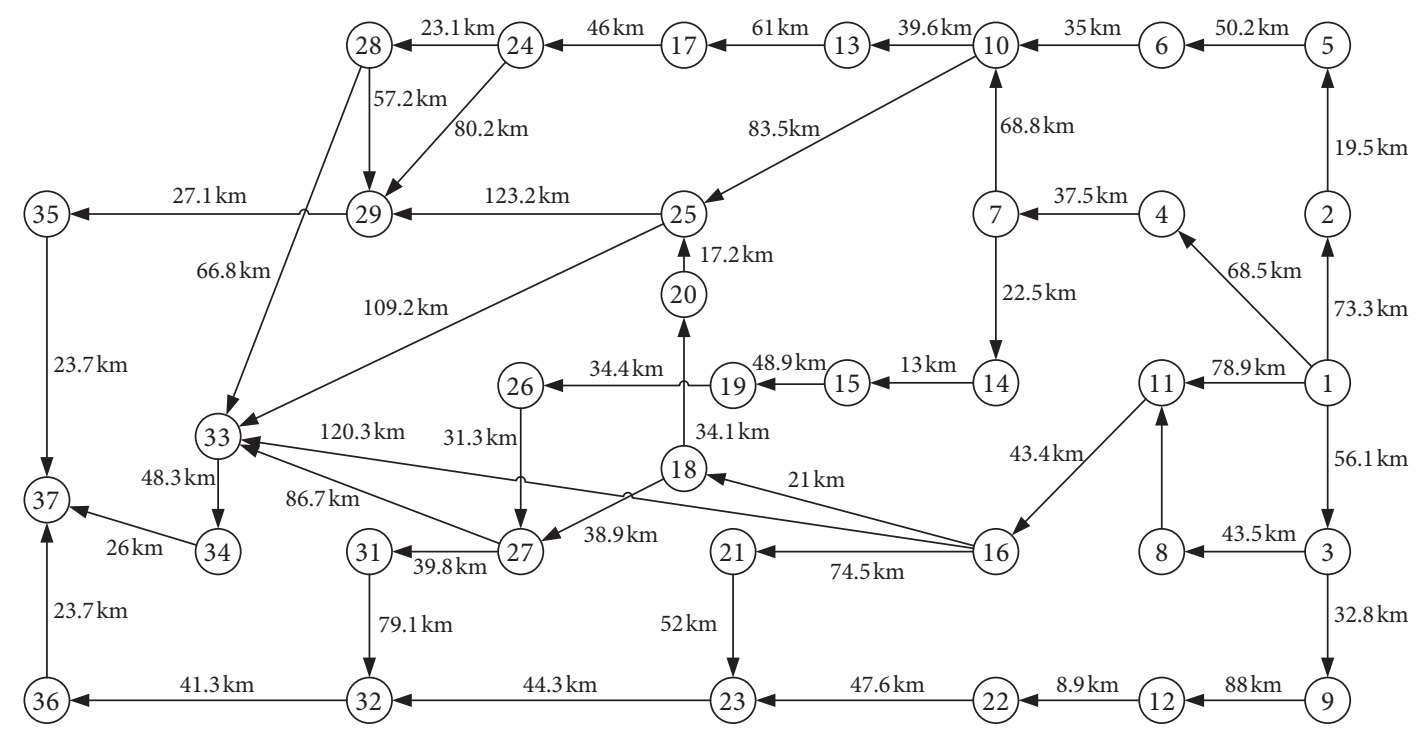

Figure 9: Freeway network topology.

TABLE 3: Sensitivity analysis parameters calibration.

\begin{tabular}{lccc}
\hline Notion & Parameters & Value & Unit \\
\hline$v_{c}$ & Urban highway running speed & 60 & $\mathrm{~km} / \mathrm{h}$ \\
$v_{h}$ & Freeway running speed & 90 & $\mathrm{~km} / \mathrm{h}$ \\
$E_{0}$ & Rated capacity of the on-board battery & 0.9 & $\mathrm{kwh}$ \\
$\eta$ & Charging efficiency & 0.8 & - \\
$S O C_{u p}$ & State of the on-board battery after charging & 120 & \\
$P_{C}$ & Charging power & 0.2 & $\mathrm{kw}$ \\
$S O C_{s}$ & Security SOC & 0.2 & - \\
$S O C_{d}$ & SOC of the destination & 4 & - \\
W & Node charging pile number & 4 & - \\
$q l_{\max }$ & Node queuing length & & \\
\hline
\end{tabular}

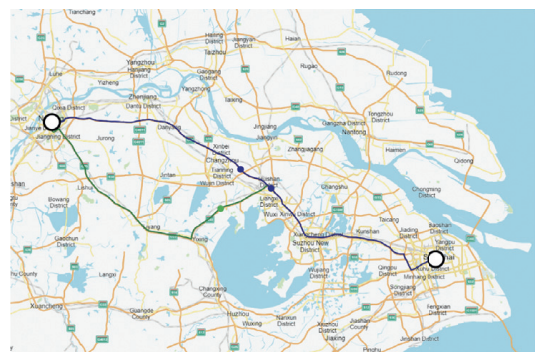

(a)

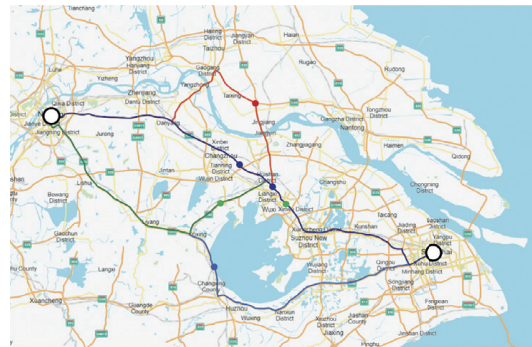

(b)

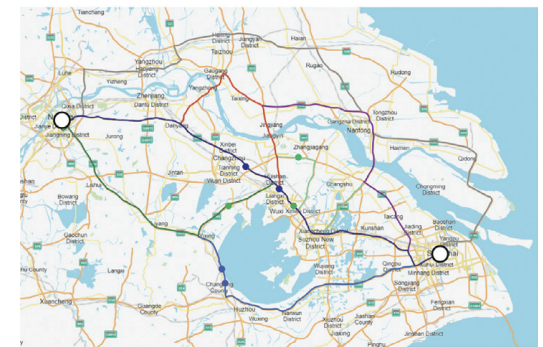

(c)

Figure 10: Effect of volume on travel paths.

It can be seen from Figure 13(a) that the average travel time of vehicles increases with the increase of volume. Under the condition of low volume, the average travel time is similar to that of mid-volume, while the average travel time of high volume increases rapidly. The reason is that charging nodes on the short travel distance path begin to reach saturation under high volume, and subsequent vehicles choose to detour the long way, resulting in a longer travel time of vehicles. As can be seen from Figure 13(b), the proportion of queuing vehicles increases with the increase of volume, and the proportion of queuing vehicles is 0 under low volume, which is because there is no need for queuing vehicles at this volume. When vehicles start to queue at medium volume, half of the vehicles are queued at high volume. It shows that volume has a great influence on the queuing of the system.

Figure 14 shows the average energy consumption of electric vehicles under low, medium, and high volumes and the corresponding change trend. It can be seen that when the volume increases, the average energy consumption of electric vehicles also increases. This is due to the increase in the number of electric vehicles in the system, and the later 


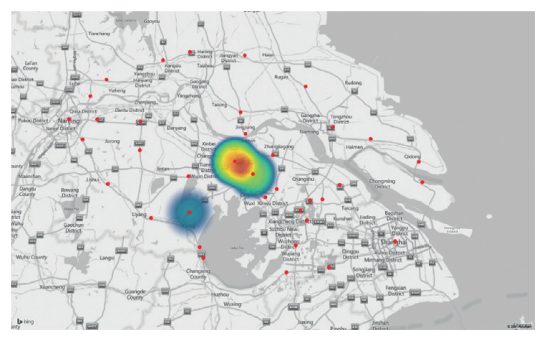

(a)

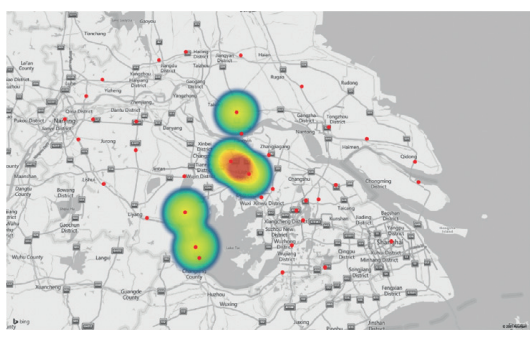

(b)

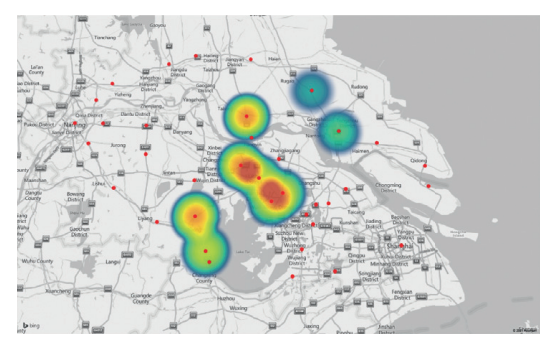

(c)

FIgURE 11: Effect of volume on node utilization.

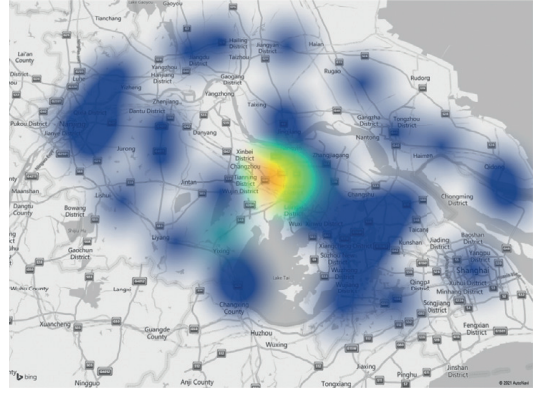

(a)

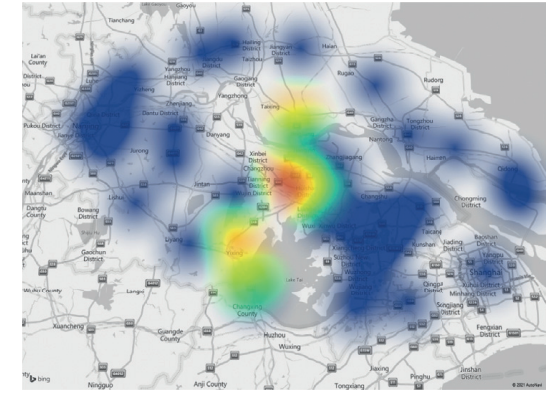

(b)

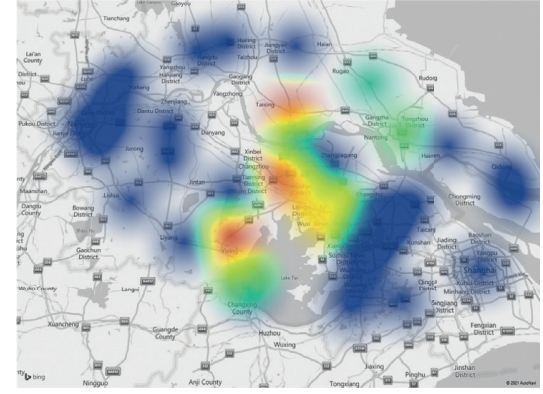

(c)

FIgURE 12: Effect of volume on utilization distribution.

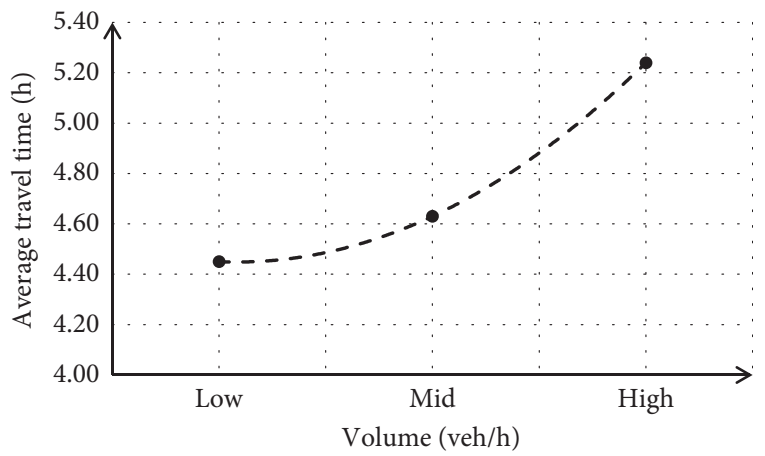

(a)

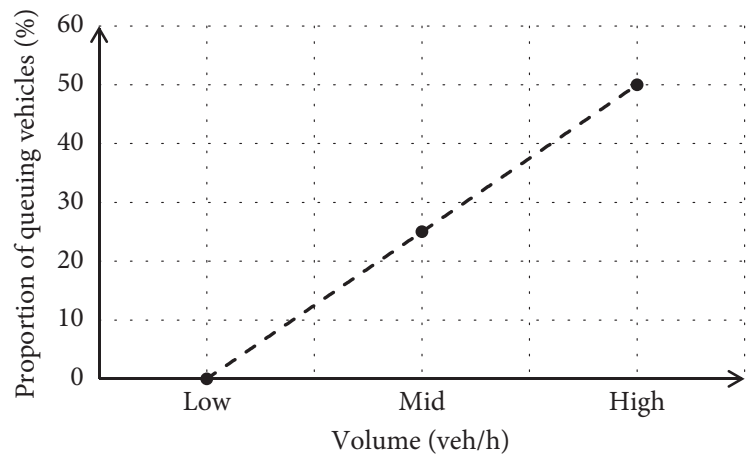

(b)

FIgURE 13: Effect of volume on traffic efficiency.

vehicles will choose a relatively uncongested path to drive, increasing the driving distance and increasing the energy consumption.

4.3. Effect of Low Temperature. Relevant studies $[35,36]$ have shown that the discharge efficiency of lithium-ion batteries is related to temperature. When the temperature drops from normal temperature $\left(25^{\circ}\right)$ to 0 degrees Celsius, the discharge efficiency of the battery drops about $15 \%$. Therefore, we choose the volume as the medium volume and analyze the travel path of the electric vehicle at low temperature when the speed is $90 \mathrm{~km} / \mathrm{h} .0^{\circ} \mathrm{C}$ is defined as the low-temperature state.

Figures 15(a) and 15(b), respectively, show the travel paths of electric vehicles under normal temperature and low temperature. It can be seen that when the temperature drops, the number of paths chosen by the vehicle will not change, but the choice of paths and charging nodes will change. The position of charging nodes at low temperature is closer to the starting point than that at normal temperature, which is because the endurance mileage of the vehicle becomes shorter due to low temperature.

Figures 16(a) and 16(b) are the thermal maps of charging node utilization under normal temperature and low temperature, respectively. There are 6 charging nodes under the normal temperature condition and only 4 charging nodes under the low-temperature condition. The problem of short endurance mileage of electric vehicles caused by low temperature has increased the service pressure in Yanqiao, Guangling, and Taihu (Huzhou) service areas.

Figures 17(a) and 17(b) show the influence of normal temperature and low temperature on the utilization distribution of charging nodes, respectively. Under the condition of 


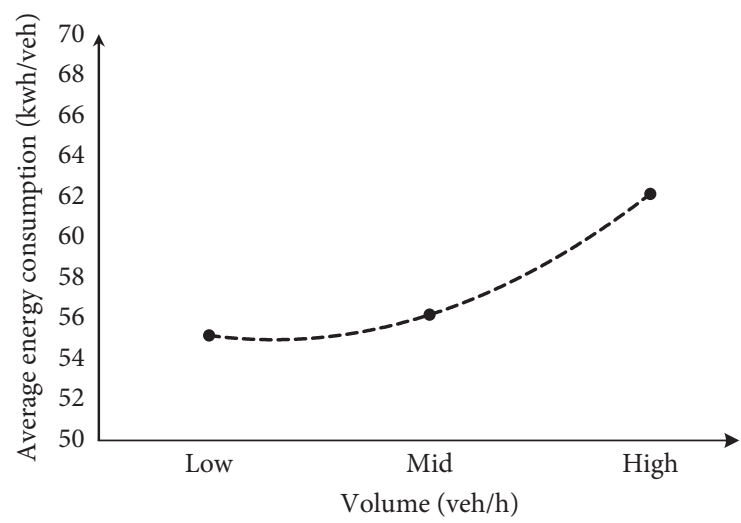

FIGURE 14: Effect of volume on energy consumption.

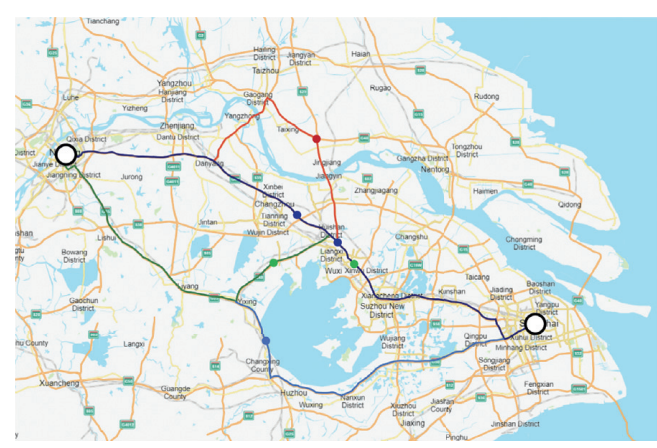

(a)

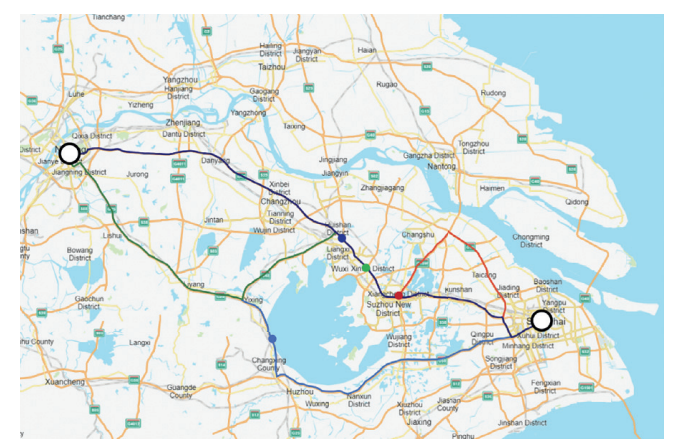

(b)

Figure 15: Effect of temperature on travel path.

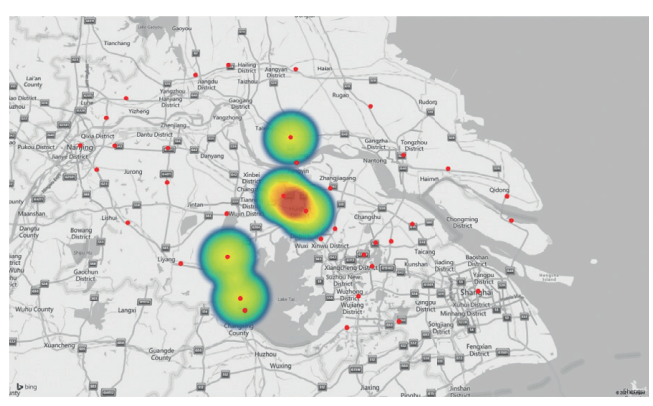

(a)

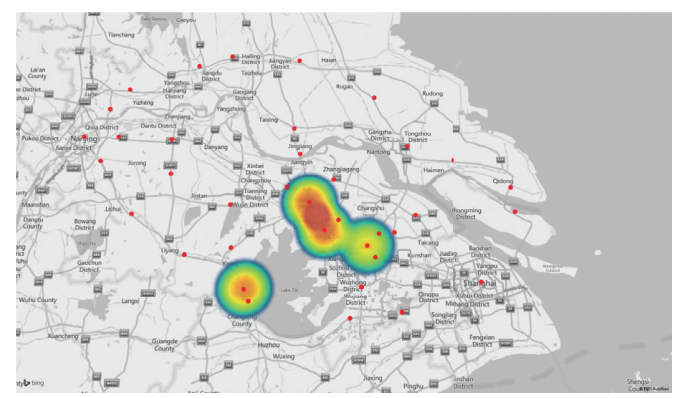

(b)

FIgURE 16: Effect of temperature on node utilization.

low temperature, the main charging nodes of electric vehicles are distributed in Wuxi and Yixing. However, charging nodes used by electric vehicles at the normal temperature are mainly distributed in Wuxi and Changzhou. In conclusion, the number of charging piles can be increased in Wuxi and Yixing areas in consideration of the reduced endurance mileage of electric vehicles under the low temperature.

Figure 18(a) shows the variation of the average travel time at different temperatures. It can be seen that temperature has no effect on the average travel time. It can be seen from Figure 18(b) that the proportion of queuing vehicles in the system at normal temperature is lower than that at low temperature, indicating that the lower the temperature is, the shorter the vehicle endurance will be, and the more queuing phenomenon will occur in the freeway network.

Figure 19 shows the average energy consumption of electric vehicles at low temperature and normal temperature. Since the temperature has little influence on the driving path, therefore, the temperature has little effect on the energy consumption of electric vehicles.

4.4. Effect of Travel Speed. According to the road safety regulations, the speed of vehicles under the freeway must be between $90 \mathrm{~km} / \mathrm{h}$ and $120 \mathrm{~km} / \mathrm{h}$. Therefore, $90 \mathrm{~km} / \mathrm{h}$, $105 \mathrm{~km} / \mathrm{h}$, and $120 \mathrm{~km} / \mathrm{h}$ are selected as the travel speed 


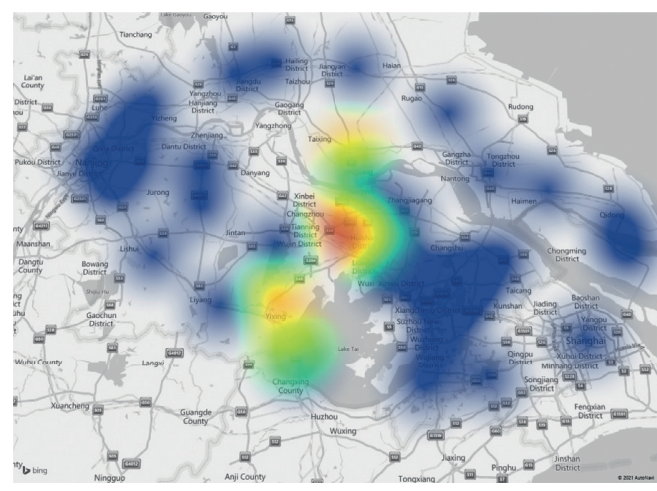

(a)

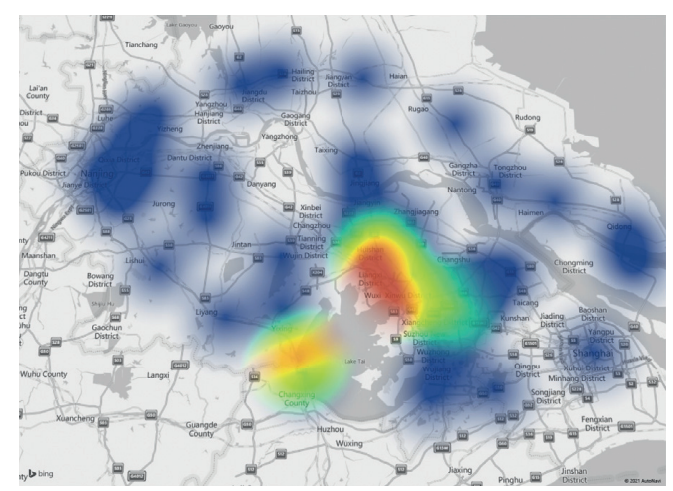

(b)

FIgURE 17: Effect of temperature on utilization distribution.

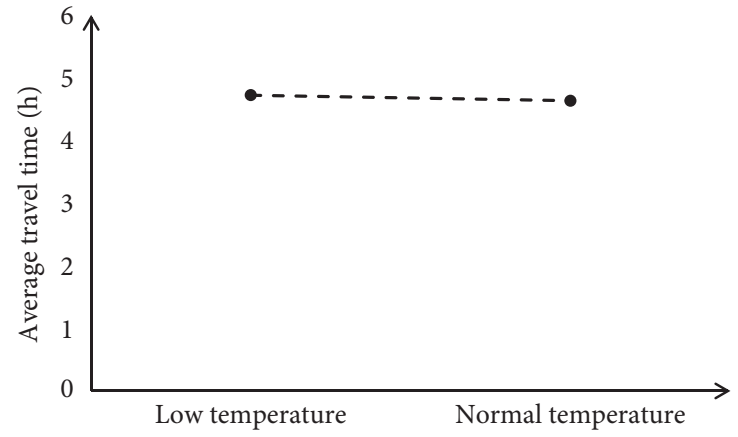

(a)

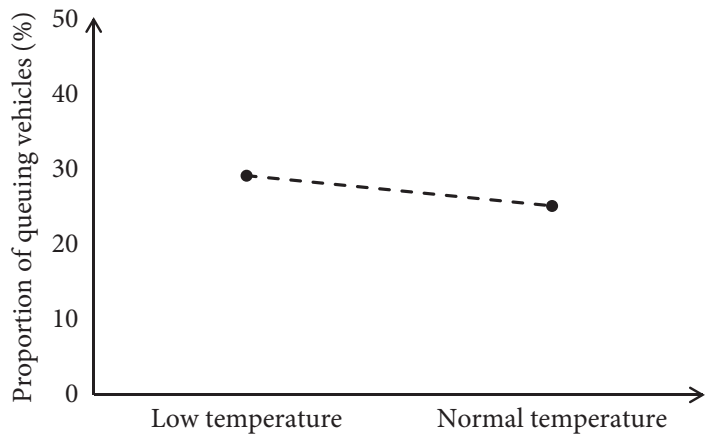

(b)

Figure 18: Effect of temperature on traffic efficiency.

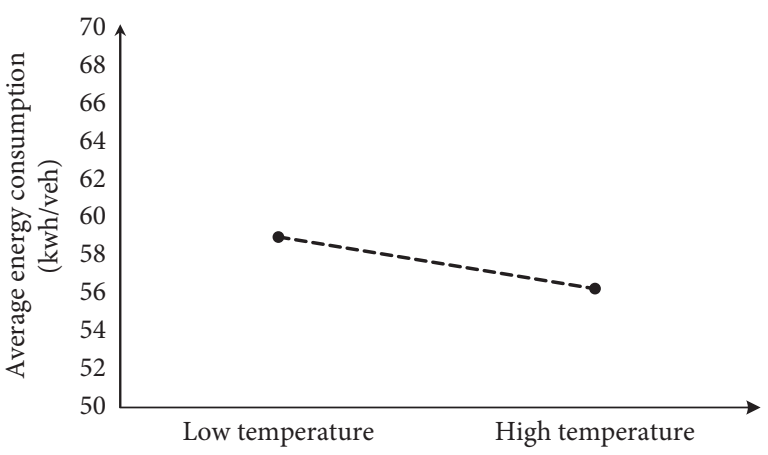

Figure 19: Effect of temperature on energy consumption.

input to analyze the influence of travel speed on the travel path of electric vehicles. Figures 20(a), 20(b), and 20(c), respectively, show the driving paths of electric vehicles with speeds of $90 \mathrm{~km} / \mathrm{h}, 105 \mathrm{~km} / \mathrm{h}$, and $120 \mathrm{~km} / \mathrm{h}$. It can be seen that, with the increase of speed, there are more paths available for vehicles in the freeway network, and the number of charging nodes available for choice also increases.

Figures 21(a), 21(b), and 21(c) are thermal maps of charging node utilization at speeds of $90 \mathrm{~km} / \mathrm{h}, 105 \mathrm{~km} / \mathrm{h}$, and $120 \mathrm{~km} / \mathrm{h}$, respectively. Combined with Figure 20, it can be seen that the Furong service area and Yanqiao service area are the main charging areas for vehicles at a speed of $90 \mathrm{~km} / \mathrm{h}$, and these two places bear the charging needs of most vehicles. When the speed increases to $105 \mathrm{~km} / \mathrm{h}$, the increase of alternative oaths brings new charging nodes for vehicles in the freeway network. At this time, the Wanshandang service area, Meicun service area, and Jiangyin Bridge service area are all in use, but Furong and Yanqiao service areas are still the charging nodes with the most serving vehicles. When the vehicle speed is $120 \mathrm{~km} / \mathrm{h}$, the increased paths make the Rugao service area and Baimi service area become the two nodes for subsequent vehicles to choose charging. At this time, the service areas of Guangling, Jiangyin Bridge, Furong, and Yanqiao are all high-utilization nodes. By comparing the experimental results at different speeds, it is shown that high speeds can improve the utilization rate of nonhot nodes.

Figures 22(a), 22(b), and 22(c) show the utilization distribution of charging nodes at $90 \mathrm{~km} / \mathrm{h}, 105 \mathrm{~km} / \mathrm{h}$, and $120 \mathrm{~km} / \mathrm{h}$, respectively. It can be concluded from Figure 22 that a faster driving speed can make more charging nodes available. It also relieves service pressure on busy nodes. The reason for the above conclusion is that faster travel speed can reduce the cost of detours (reduce the detour time), thus increasing the number of detour vehicles.

Figure 23(a) shows a negative correlation between travel time and travel speed. Combined with Figure 23(b), it can be concluded that electric vehicles reduce travel time by reducing queuing time. 


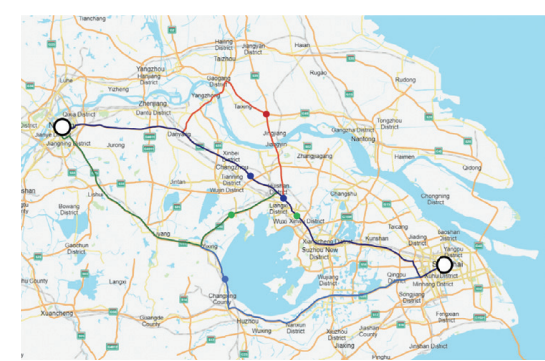

(a)

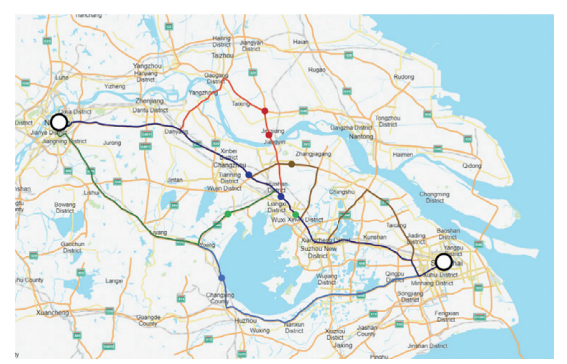

(b)

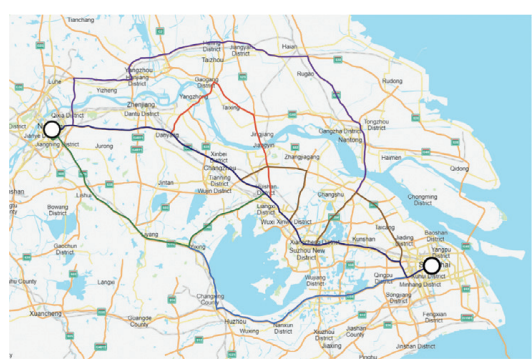

(c)

FIgURE 20: Effect of travel speed on travel paths.

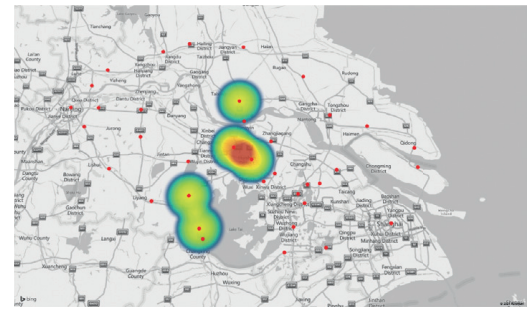

(a)

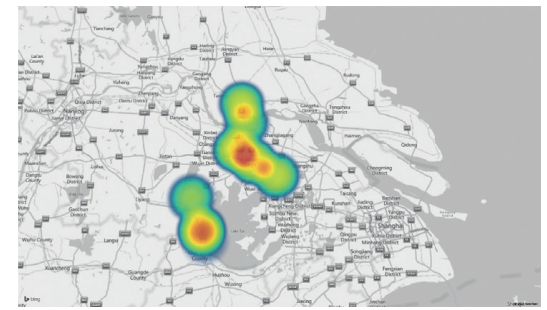

(b)

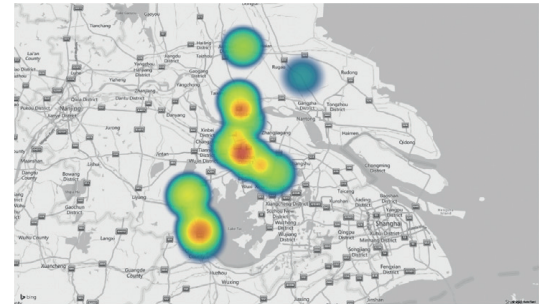

(c)

Figure 21: Effect of travel speed on node utilization.

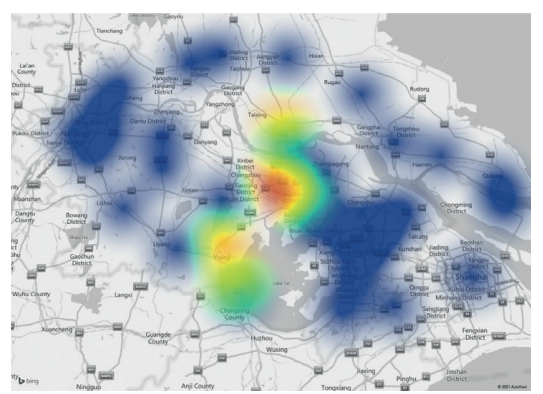

(a)

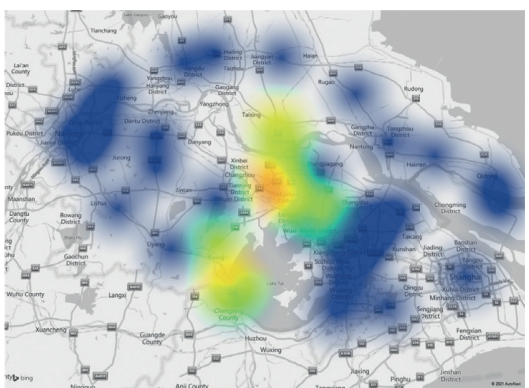

(b)

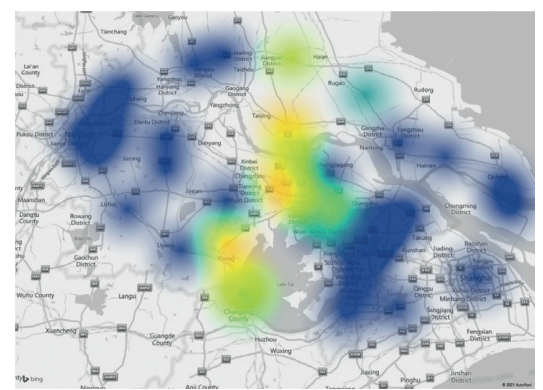

(c)

FIGURE 22: Effect of travel speed on utilization distribution.

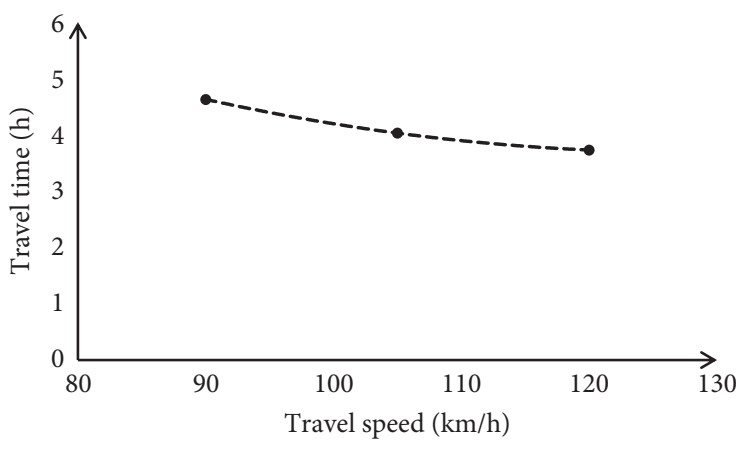

(a)

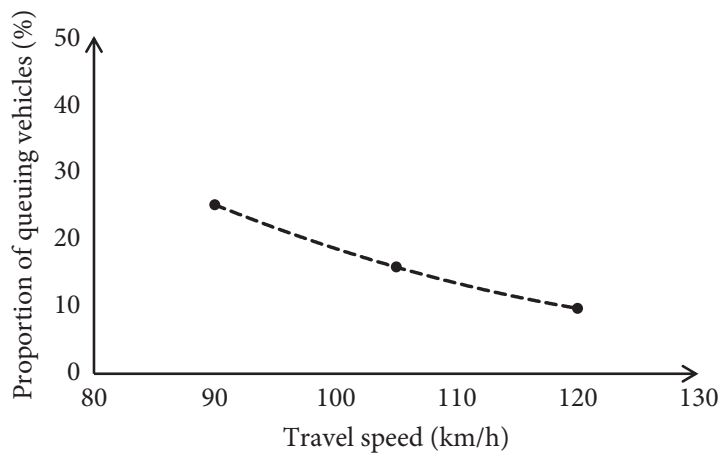

(b)

FiguRE 23: Effect of travel speed on traffic efficiency.

Figure 24 shows the average energy consumption and its change trend of electric vehicles at $90 \mathrm{~km} / \mathrm{h}, 105 \mathrm{~km} / \mathrm{h}$, and $120 \mathrm{~km} / \mathrm{h}$. Combined with the above analysis, a faster travel speed will lead electric vehicles to choose a longer path. Combined with the experimental results of Ray Galvin's model, it can be seen that travel speed has a significant 


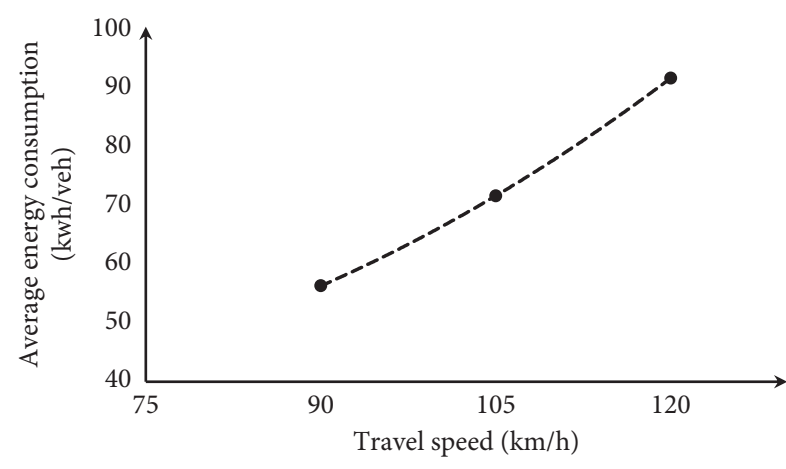

FIgURE 24: Effect of travel speed on energy consumption.

impact on energy consumption. The faster the travel speed, the greater the average energy consumption of vehicles in the system.

\section{Conclusions}

In this paper, electric vehicles are selected as the research object, the charging problem of electric vehicles in the process of driving on freeways is considered, and the charging strategy and path planning of electric vehicles under the freeway network are studied. The research conclusions of this paper are as follows:

(1) Electric vehicle path planning model is presented in this paper, and through the case analysis, the proposed method can improve the efficiency of electric vehicles driving on the highway.

(2) This paper analyzes the influence of traffic volume on the driving path of electric vehicles and the state of the freeway network. The results show that the traffic volume, temperature, and travel speed have an impact on the electric vehicle travel path. The number of paths available for vehicles and the number of charging nodes used will increase with the increase of volume, but the increase of volume will lead to the increase of average travel time, and the proportion of queuing vehicles will gradually increase.

(3) Considering that temperature has a great influence on battery efficiency, the influence of temperature on the driving paths of electric vehicles and the state of the freeway network was analyzed to explore the model effect under low temperature in winter. Temperature variation has a great influence on the selection of charging nodes.

(4) This paper also analyzes the influence of travel speed on the path of electric vehicles and the state of the freeway network. The results show that, with the increase of speed, the travel time of vehicles decreases, the number of alternative paths and charging nodes increases, and the proportion of queuing vehicles decreases.

(5) From the perspective of the road network, it can be seen that Furong service area, Yanqiao service area, Meicun service area, Yixing service area, and
Guangling service area are the main charging nodes selected. These nodes are all located in Changzhou, Wuxi. It can be seen that electric vehicles from Shanghai to Nanjing generally need to be charged here, and charging piles can be added here if necessary in the future.

The further expansion of this paper can start from the perspective of the system, optimize the system with the highest system efficiency as the objective, and enrich the types of road network information (such as charging fees, tolls) to improve the research. From the perspective of research methods, machine learning is a popular method in the field of transportation. At the same time, there have been many research results in the field of batteries. Liu et al. [37] proposed a classification method based on a random forest to quantify the characteristics of battery manufacturing. Liu et al. [38] proposed a machine learning framework based on Gaussian process regression, which can quantify the importance of four intermediate product characteristic variables and analyze their impact on battery electrode mass load forecasting. Therefore, in future research, we will consider the method of machine learning to explore its application in path planning.

\section{Data Availability}

The data used to support the findings of this study are available from the corresponding author upon request.

\section{Conflicts of Interest}

The authors declare that there are no conflicts of interest regarding the publication of this paper.

\section{Acknowledgments}

The research was supported by the National Natural Science of China (71801042 and 51878166) and the Natural Science of Jiangsu Province (BK20180381).

\section{References}

[1] K. N. Genikomsakis and G. Mitrentsis, "A computationally efficient simulation model for estimating energy consumption of electric vehicles in the context of route planning applications," Transportation Research Part D: Transport and Environment, vol. 50, pp. 98-118, 2017.

[2] X. Xu, H. M. A. Aziz, and R. Guensler, "A modal-based approach for estimating electric vehicle energy consumption in transportation networks," Transportation Research Part D: Transport and Environment, vol. 75, pp. 249-264, 2019.

[3] Z. Yi, J. Smart, and M. Shirk, "Energy impact evaluation for eco-routing and charging of autonomous electric vehicle fleet: ambient temperature consideration," Transportation Research Part C: Emerging Technologies, vol. 89, pp. 344-363, 2018.

[4] G. Wager, J. Whale, and T. Braunl, "Driving electric vehicles at highway speeds: the effect of higher driving speeds on energy consumption and driving range for electric vehicles in Australia," Renewable and Sustainable Energy Reviews, vol. 63, pp. 158-165, 2016. 
[5] R. Zhang and E. Yao, "Electric vehicles' energy consumption estimation with real driving condition data," Transportation Research Part D: Transport and Environment, vol. 41, pp. 177-187, 2015.

[6] C. De Cauwer, J. Van Mierlo, and T. Coosemans, "Energy consumption prediction for electric vehicles based on realworld data," Energies, vol. 8, no. 8, pp. 8573-8593, 2015.

[7] A. I. Croce, G. Musolino, C. Rindone, and A. Vitetta, "Energy consumption of electric vehicles: models' estimation using big data (FCD)," Transportation Research Procedia, vol. 47, pp. 211-218, 2020.

[8] A. I. Croce, G. Musolino, C. Rindone, and A. Vitetta, "Sustainable mobility and energy resources: a quantitative assessment of transport services with electrical vehicles," Renewable and Sustainable Energy Reviews, vol. 113, Article ID 109236, 2019.

[9] K. Liu, C. Zou, K. Li, and T. Wik, "Charging pattern optimization for lithium-ion batteries with an electrothermalaging model," Ieee Transactions on Industrial Informatics, vol. 14, no. 12, pp. 5463-5474, 2018.

[10] K. Liu, X. Hu, Z. Yang, Y. Xie, and S. Feng, "Lithium-ion battery charging management considering economic costs of electrical energy loss and battery degradation," Energy Conversion and Management, vol. 195, pp. 167-179, 2019.

[11] K. Liu, K. Li, H. Ma, J. Zhang, and Q. Peng, "Multi-objective optimization of charging patterns for lithium-ion battery management," Energy Conversion and Management, vol. 159, pp. 151-162, 2018.

[12] M. Paschero, G. L. Storti, A. Rizzi, F. M. F. Mascioli, and G. Rizzoni, "A novel mechanical analogy-based battery model for SoC estimation using a multicell EKF," Ieee Transactions on Sustainable Energy, vol. 7, no. 4, pp. 1695-1702, 2016.

[13] X. Dong, C. Zhang, and J. Jiang, Evaluation of SOC Estimation Method Based on EKF/AEKF under Noise Interference, H. Wang, X. Wang, J. Yan, J. Wu, Y. Yang, and H. Li, Eds., pp. 520-525, Cleaner Energy for Cleaner Cities, Salt Lake City, USA, 2018.

[14] J. Li, J. Klee Barillas, C. Guenther, and M. A. Danzer, "Sequential Monte Carlo filter for state estimation of LiFePO 4 batteries based on an online updated model," Journal of Power Sources, vol. 247, pp. 156-162, 2014.

[15] D. Baek, Y. Chen, A. Bocca et al., "Battery-aware operation range estimation for terrestrial and aerial electric vehicles," IEEE Transactions on Vehicular Technology, vol. 68, no. 6, pp. 5471-5482, 2019.

[16] D. Baek, Y. K. Chen, and N. Chang, "Battery-aware electric truck delivery route exploration," Energies, vol. 13, no. 8, 2020.

[17] Y. Chen, D. Baek, J. Kim et al., "A SystemC-AMS framework for the design and simulation of energy management in electric vehicles," Ieee Access, vol. 7, pp. 25779-25791, 2019.

[18] O. Arslan and O. E. Karaşan, "A Benders decomposition approach for the charging station location problem with plugin hybrid electric vehicles," Transportation Research Part B: Methodological, vol. 93, pp. 670-695, 2016.

[19] F. Wu and R. Sioshansi, "A stochastic flow-capturing model to optimize the location of fast-charging stations with uncertain electric vehicle flows," Transportation Research Part D: Transport and Environment, vol. 53, pp. 354-376, 2017.

[20] J. He, H. Yang, T.-Q. Tang, and H.-J. Huang, "An optimal charging station location model with the consideration of electric vehicle's driving range," Transportation Research Part C: Emerging Technologies, vol. 86, pp. 641-654, 2018.

[21] F. He, Y. Yin, and J. Zhou, "Deploying public charging stations for electric vehicles on urban road networks,"
Transportation Research Part C: Emerging Technologies, vol. 60, pp. 227-240, 2015.

[22] M. Kchaou Boujelben and C. Gicquel, "Efficient solution approaches for locating electric vehicle fast charging stations under driving range uncertainty," Computers \& Operations Research, vol. 109, pp. 288-299, 2019.

[23] R. Chen, X. Qian, and L. Miao, "Optimal charging facility location and capacity for electric vehicles considering route choice and charging time equilibrium[J]," Computers \& Operations Research, vol. 113, 2020.

[24] W. Kong, Y. Luo, and G. Feng, "Optimal location planning method of fast charging station for electric vehicles considering operators, drivers, vehicles, traffic flow and power grid," Energy, vol. 186, 2019.

[25] M. Schücking, P. Jochem, W. Fichtner, O. Wollersheim, and K. Stella, "Charging strategies for economic operations of electric vehicles in commercial applications," Transportation Research Part D: Transport and Environment, vol. 51, pp. 173-189, 2017.

[26] X. Dong, Y. Mu, X. Xu et al., “A charging pricing strategy of electric vehicle fast charging stations for the voltage control of electricity distribution networks," Applied Energy, vol. 225, pp. 857-868, 2018.

[27] Z. Yi and M. Shirk, "Data-driven optimal charging decision making for connected and automated electric vehicles: a personal usage scenario," Transportation Research Part C: Emerging Technologies, vol. 86, pp. 37-58, 2018.

[28] M. Elgarej, M. Khalifa, and M. Youssfi, "Optimized path planning for electric vehicle routing and charging station navigation systems," International Journal of Applied Metaheuristic Computing, vol. 11, no. 3, pp. 58-78, 2020.

[29] F. Guo, Z. H. Huang, and W. L. Huang, "Integrated location and routing planning of electric vehicle service stations based on users' differentiated perception under a time-sharing leasing mode," Journal of Cleaner Production, vol. 277, p. 22, 2020.

[30] C. Wang, F. He, X. Lin, Z.-J. M. Shen, and M. Li, “Designing locations and capacities for charging stations to support intercity travel of electric vehicles: an expanded network approach," Transportation Research Part C: Emerging Technologies, vol. 102, pp. 210-232, 2019.

[31] H. Liu, W. Yin, and X. Yuan, "Reserving charging decisionmaking model and route plan for electric vehicles considering information of traffic and charging station," Sustainability, vol. 10, no. 5, 2018.

[32] Z. Li, K. Dey, M. Chowdhury, and P. Bhavsar, "Connectivity supported dynamic routing of electric vehicles in an inductively coupled power transfer environment," IET Intelligent Transport Systems, vol. 10, no. 5, pp. 370-377, 2016.

[33] Y. Wang, J. Bi, and C. Lu, "Route guidance strategies for electric vehicles by considering stochastic charging demands in a time-varying road network," Energies, vol. 13, no. 9, 2020.

[34] R. Galvin, "Energy consumption effects of speed and acceleration in electric vehicles: laboratory case studies and implications for drivers and policymakers," Transportation Research Part D: Transport and Environment, vol. 53, pp. 234-248, 2017.

[35] C. Vidal, O. Gross, R. Gu, P. Kollmeyer, and A. Emadi, "xEV Li-ion battery low-temperature effects-review," IEEE Transactions on Vehicular Technology, vol. 68, no. 5, pp. 4560-4572, 2019.

[36] Y. Ji, Y. Zhang, and C.-Y. Wang, "Li-ion cell operation at low temperatures," Journal of the Electrochemical Society, vol. 160, no. 4, pp. A636-A649, 2013. 
[37] K. Liu, X. Hu, and H. Zhou, "Feature analyses and modelling of lithium-ion batteries manufacturing based on random forest classification," Medicine, vol. 20, 2021.

[38] K. L. Liu, Z. B. Wei, and Z. L. Yang, "Mass load prediction for lithium-ion battery electrode clean production: a machine learning approach," Journal of Cleaner Production, vol. 289, 2021. 